ORIGINAL ARTICLE

\title{
Words matter: increasing the implementation of clinical guidelines
}

\section{S Michie, K Lester}

Qual Saf Health Care 2005;14:367-370. doi: 10.1136/qshc.2005.014100

See end of article for authors' affiliations

.....................

Correspondence to: Professor S Michie, Centre for Outcomes Research and Effectiveness, University College London, 1-19 Torrington Place, London WCIE 7HB, UK; s.michie@ucl.ac.uk

Accepted for publication 15 August 2005
Objectives: To determine whether writing clinical guideline recommendations in behaviourally specified "plain English" language increases the likelihood of their implementation by service users (patients). Design: Randomised controlled trial in which participants received either the original text of the National Institute for Clinical Excellence (NICE) public guidelines for the management of schizophrenia or a behaviourally specified text with the same content.

Setting: Mental health service user networks and voluntary sector organisations within two inner London boroughs.

Participants: Eighty four mental health service users recruited by post or face to face contact at service user meetings.

Intervention: The section of the NICE public guidelines for schizophrenia concerning psychological and pharmacological treatments was rewritten to improve style and behavioural specificity by applying evidence-based and psychologically informed principles of good written communication.

Outcome measures: Cognitive predictors of behaviour, as specified by the evidence based theory of planned behaviour, constituted the primary outcome as it was not possible to measure the actual behaviour of guideline implementation. The predictors were behavioural intentions to implement the guidelines, attitudes towards implementation, and perceived behavioural control over implementation. Satisfaction with the guidelines and perceived comprehension were also measured.

Results: Behaviourally specified "plain English" guidelines led to stronger intentions to implement the guidelines, more positive attitudes towards them, and greater perceived behavioural control over using them. There was no difference in satisfaction or perceived comprehension.

Conclusions: Writing guidelines with high behavioural specificity in conjunction with the use of "plain English" may be a simple and effective method of increasing their implementation. Evaluation with a behavioural outcome is now needed.
W hile evidence-based clinical guidelines have the potential to improve the quality of patient care, there has been limited success in encouraging healthcare professionals to implement them. ${ }^{1}$ There is increasing focus on the role of patients in facilitating implementation, evidenced by the production of public versions of guidelines in the form of patient information leaflets, audiotapes, and videos.

The first clinical guideline produced by the UK's National Institute for Clinical Excellence was on schizophrenia. A lay version, known as Information for the Public (IFP), was also produced: "Treating and managing schizophrenia (core interventions): understanding NICE guidance-information for people with schizophrenia, their advocates and carers, and the public". ${ }^{2}$ It aims to provide service users, carers, and the public with evidencebased recommendations on the best available psychological and pharmacological treatments. Writing guidelines in a suitable style and format for patients and the public is considered to be important for effective implementation, ${ }^{3}$ as is writing recommendations that are behaviourally specific. ${ }^{4}$ This is supported by evidence from Grol and colleagues ${ }^{5}$ who compared the implementation by clinicians of guidelines that were defined precisely and clearly with those that were vague and non-specific: the former were implemented on $67 \%$ of occasions and the latter on just $36 \%$ of occasions.

Presenting evidence and recommendations in a clear, concise, accessible, and flexible format facilitates the retrieval and assimilation of specific information. ${ }^{2}{ }^{6}$ Specification in terms of how, what, when, where, and why improves both comprehension and recall ${ }^{78}$ and planning and behaviour. ${ }^{9}$
According to Grol and colleagues, ${ }^{5}$ behaviourally specific clinical guidelines should provide a concrete and precise description of desired performance, giving detailed advice on which performance is appropriate in which situation and in what patient group and determining which factors or conditions should be taken into account. Behaviour change is more likely to occur following a specific behavioural plan $^{10}{ }^{11}$ and when the target behaviour is defined in specific and concrete terms. ${ }^{12}$

This randomised controlled trial investigates the impact of increased behavioural specificity and use of "plain English"* in writing guideline recommendations on (1) cognitive predictors of behaviour and (2) satisfaction and perceived comprehension. ${ }^{13}$ The behaviour of interest is the extent to which the evidence-based information and recommendations in the public guideline is used by mental health service users. Since behaviour could not be studied directly, cognitions that have been shown to predict behaviour (attitudes towards the behaviour, perceived control over the behaviour, and intention to perform the behaviour) form the primary outcome measures. These cognitions are component constructs of the theory of planned behaviour. ${ }^{14}$

The theory of planned behaviour is a motivational theory of behaviour as it assumes that intention to perform a behaviour is its most important determinant. The strength

* Plain English involves the production of public information in a form that the intended audience can read, understand, and act upon the first time it is read and which takes into account language, design, and layout. 
of intention is determined by three variables; whether the person is in favour of performing the behaviour ("attitude"), how much social pressure to do it that they feel ("subjective norm"), and whether they feel in control of performing the behaviour ("perceived behavioural control"). Generally, the more positive people's attitudes and subjective norms regarding the behaviour and the greater their perceived behavioural control, the stronger their intention to perform the behaviour. Attitude is composed of beliefs about the outcomes of the behaviour and evaluations of those outcomes; subjective norm is composed of beliefs about how others would like them to behave and their evaluation of these beliefs; and perceived behaviour control is composed of how much control they have over the behaviour and their confidence in performing the behaviour.

It is hypothesised that increased behavioural specificity and use of "plain English" is associated with (1) greater behavioural intentions to implement the guidelines; (2) more positive attitudes toward the behaviour; (3) greater perceived behavioural control to implement the guideline; (4) greater satisfaction with the guidelines; and (5) greater perceived comprehension of the guidelines. The theory of planned behaviour is partially tested by investigating whether attitudes towards the behaviour and perceived behavioural control predict behavioural intentions to implement the guidelines.

\section{METHODS \\ Design}

Participants were assigned independently within a blind randomised controlled design to the original or intervention text condition using a random number table, with the constraint of equal sample sizes assigned to each text condition.

\section{Participants}

Eighty four mental health service users from two inner London boroughs were recruited via service user networks and voluntary sector organisations. The only exclusion criterion was insufficient English to understand the study materials. Age range was normally distributed with a median range of $41-50$ years; $51 \%$ of the sample were women. $75 \%$ of the sample classified themselves as white British, $5 \%$ as white Irish, 5\% from the Indian subcontinent, $2 \%$ black, $6 \%$ mixed white/black/Asian, 2\% Chinese, and the rest "other" ethnic groups.

Study power, calculated retrospectively, was 0.57 for behavioural intentions, 0.89 for attitudes towards the behaviour, and 0.96 for perceived behavioural control, showing that the study was powered to detect medium or large effects. Target power was calculated on the basis of a medium effect size with a sample of 102 participants.

Participants were invited to participate in the study by post $(\mathrm{n}=300)$ or at meetings of mental health service users $(\mathrm{n}=100)$. Random assignment occurred before consent to participate. Of the 87 responses returned, 84 were completed (42 in each condition). This response rate of $22 \%$ is typical for this population: the Local Health Services Questionnaire $(2003)^{15}$ conducted in the same part of London had a response rate of $28 \%$.

\section{Procedure}

Ethics approval for the study was obtained from Camden \& Islington Community Health Service local research ethics committee. Consent was obtained before the start of the study for all participants. After consenting, participants were asked to read the study text leaflet and complete the questionnaire from the point of view of someone with schizophrenia.
Table 1 Excerpts of text taken from original NICE guidelines in comparison with behaviourally specific "plain English" text

\begin{tabular}{|c|c|}
\hline Original text & Behaviourally specific text \\
\hline $\begin{array}{l}\text { Also, if you want psychological } \\
\text { help, you will need antipsychotic } \\
\text { medicines at the same time. } \\
\text { Remember, the decision about } \\
\text { which medicine to take is best } \\
\text { made by you and your doctors } \\
\text { together. }\end{array}$ & $\begin{array}{l}\text { You will need to take these medicines } \\
\text { if you also want psychological help. } \\
\text { You should decide which medicine is } \\
\text { best for you with the help of your } \\
\text { doctors. }\end{array}$ \\
\hline $\begin{array}{l}\text { It is also particularly useful if } \\
\text { you have symptoms that won't } \\
\text { go away and are troubling to } \\
\text { you. CBT is also helpful if you } \\
\text { are having trouble accepting } \\
\text { that you have schizophrenia } \\
\text { (sometimes called "lack of } \\
\text { insight"), and it may help if } \\
\text { you tend not to take your } \\
\text { medicine properly (sometimes } \\
\text { called "poor compliance") }\end{array}$ & $\begin{array}{l}\text { This is particularly useful if you: } \\
\text { - have symptoms that won't go } \\
\text { away and are troubling you; } \\
\text { are having trouble accepting that } \\
\text { you have schizophrenia } \\
\text { (sometimes called "lack of } \\
\text { insight"); or } \\
\text { tend not to take your medicine } \\
\text { properly (sometimes called "poor } \\
\text { compliance"). }\end{array}$ \\
\hline
\end{tabular}

\section{Development of the intervention text}

The sections concerning psychological and medical treatments from the guideline "Treating and managing schizophrenia (core interventions): understanding NICE guidance-information for people with schizophrenia, their advocates and carers, and the public" were rewritten by applying good practice recommendations for producing written information. ${ }^{16}$ The text was amended to increase behavioural specificity, personalise the message, avoid highly technical language, use active rather than passive verbs, avoid negative or alarmist approaches, and to include appropriate numerical information. Content, layout, and presentation remained constant across versions, although the amended text used bullet points, headings, and boxes with key points (see examples in table 1).

Independent raters using a reliable coding frame (Cohen's kappa 0.67-0.81) found the intervention text to be more behaviourally specific $\left(\chi^{2}(1)=8.673, \mathrm{p}<0.001, \Phi_{\mathrm{c}}=0.329\right)$ and as personalising the message more often than the standard text $\left(\chi^{2}(1)=3.532, \mathrm{p}<0.05\right.$, ФС $\left.=0.187\right)$.

\section{Outcome measures}

A questionnaire (available from the first author) measured three cognitive predictors of behaviour specified by the theory of planned behaviour: ${ }^{14}$ intention, attitude, and perceived behavioural control (fig 1). Subjective norm was not measured as it is primarily of importance where the behaviour directly affects the health of a significant other or when the behaviour is performed publicly. ${ }^{17}$ Perceived

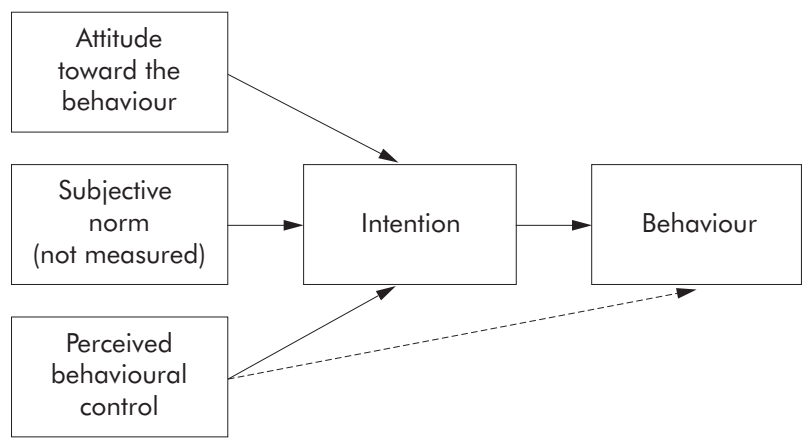

Figure 1 Schematic representation of the four component predictors of behaviour in the theory of planned behaviour. 
comprehension and satisfaction were also measured. Measures were reliable: behavioural intentions (three items, alpha $=0.93$ ), attitude toward the behaviour (five items, alpha $=0.95$ ), perceived behavioural control (four items, alpha $=0.70$ ), satisfaction (five items, alpha $=0.81$ ), and perceived comprehension (three items, alpha $=0.64$ ). Respondents were asked to respond from the point of view of someone with schizophrenia on 5 point scales. Example items include:

\section{(i) Behavioural intention}

I intend to use the information given in the leaflet when/if I make choices about my treatment in the future ... (extremely unlikely-extremely likely)

\section{(ii) Attitude toward the behaviour}

For me to use the information given in the leaflet when/if I make choices about my treatment in the future would be ... (valuable-worthless, good-bad, harmful-beneficial, relevant-irrelevant, welcome-unwelcome)

\section{(iii) Perceived behavioural control}

For me to make decisions about treatment options after reading the leaflet would be ... (impossible-possible)

\section{(iv) Satisfaction}

This leaflet is very good at explaining the reasons for different treatments ... (strongly disagree-strongly agree)

\section{(v) Perceived comprehension}

After having read the leaflet, I understand a great deal more about possible treatments for schizophrenia ... (strongly disagree-strongly agree)

\section{Analysis of data}

One tailed independent sample $t$ tests and Cohen's $d$ criteria ${ }^{18}$ were used to investigate differences between conditions. Nine missing responses were imputed using the regression estimation function. ${ }^{19}$ A standard multiple regression analysis was used to assess the relationship between intention (the dependent variable in the model) and attitude and perceived behavioural control (the independent variables). This allows an estimate of the percentage of variance of intention that is predicted by the independent variables and the relative importance of the independent variables in the equation. Two multivariate outlying values were omitted due to standardised residuals more than three standard deviations away from the predicted value. Normality of the distribution was assumed if measures of skewness and kurtosis fell within the acceptable range of $+1 /-1 .{ }^{20}$ Moderately negatively skewed data were corrected using a reflexive transformation. However, this transformation made no significant difference to either the value of the test statistic or related significance level, so the untransformed statistics are presented.

\section{RESULTS}

\section{Cognitive predictors of behaviour}

As shown in table 2, behavioural intentions were stronger for the intervention text $(3.71 \vee 3.23, t(82)=2.161, \mathrm{p}<0.05$, $d=0.47)$, attitudes toward the behaviour were more positive $(4.26 v 3.64, t(75.816)=3.207, \mathrm{p}<0.01$ (WelchSatterthwaite corrected degrees of freedom), $d=0.70$ ), and perceived behavioural control was greater (3.91 $v 3.33$, $t(82)=3.739, \mathrm{p}<0.01, d=0.82)$. These are medium to large effects.

\section{Satisfaction and perceived comprehension}

There was no improvement in the intervention text in satisfaction (3.65 $v 3.42$ ) or in perceived comprehension (3.91 $v 3.76)$.

\section{Test of the behavioural model}

Attitudes toward the behaviour and perceived behavioural control significantly predicted behavioural intention ( $\mathrm{F}(2$, $79)=56.875, \mathrm{p}<0.01$ ) with the full model accounting for $59.0 \%$ of the variance. Partial regression coefficients are significant for both attitudes and perceived behavioural control, with attitudes making a greater contribution to the prediction of behavioural intentions than perceived behavioural control $(t(79)=5.084, \mathrm{p}<0.01$ and $t(79)=3.795$, $\mathrm{p}<0.01$, respectively, and $\beta$ coefficients 0.483 and 0.360 , respectively).

\section{DISCUSSION}

Rewriting sections of the NICE public guidelines for schizophrenia in behaviourally specified "plain English" language strengthened the cognitive determinants of implementation behaviour among mental health service users. Simple amendments to the style and specificity of the guidelines led to a strengthening of perceived behavioural control, more positive attitudes towards the use of the guidelines, and stronger behavioural intentions to implement the guidelines. The validity of the theory of planned behaviour model is supported: attitudes and perceived behavioural control predicted $59 \%$ of the variance in intentions, which is higher than is generally achieved in health behaviour research. ${ }^{21}$

This study illustrates the importance of not only disseminating evidence-based recommendations through guidelines, but also ensuring that they are written in a style that will maximise the likelihood of their implementation. Defining guideline recommendations in specific concrete terms and presenting them in a style that optimises implementation provides a greater clarity about what is required by clinicians and service users, and a greater certainty about whether implementation has been accomplished. Specific concrete statements are more likely to be understood, remembered, and acted upon, and can serve as benchmarks or "performance indicators" in a way that many current guideline recommendations cannot.

Table 2 Mean (SD) values and test statistics for cognitive predictors as a function of text version $(n=42)$

\begin{tabular}{|c|c|c|c|c|c|}
\hline Scale (end points) & Intervention text & Original text & $\begin{array}{l}\text { Difference } \\
(95 \% \mathrm{Cl})\end{array}$ & $\begin{array}{l}\text { Effect size } \\
\text { (Cohen's } d \text { ) }\end{array}$ & $\begin{array}{l}t \text { value } \\
\text { (df }=82 \text { ) }\end{array}$ \\
\hline Intention (1 least, 5 most) & $3.71(0.97)$ & $3.23(1.08)$ & 0.48 (0.04 to 0.93$)$ & 0.47 & $2.161^{*}$ \\
\hline $\begin{array}{l}\text { Attitude toward the behaviour } \\
\text { (1 least positive, } 5 \text { most positive) }\end{array}$ & $4.26(0.75)$ & $3.64(1.00)$ & $0.62(0.24$ to 1.00$)$ & 0.70 & $3.207^{* *}$ \\
\hline $\begin{array}{l}\text { Perceived behavioural control } \\
\text { (1 least, } 5 \text { most) }\end{array}$ & $3.91(0.67)$ & $3.33(0.74)$ & 0.58 (0.27 to 0.88$)$ & 0.82 & $3.739^{* *}$ \\
\hline
\end{tabular}

Higher scores indicate stronger intentions, more positive attitudes and increased perceived behavioural control. ${ }^{*} p<0.05 ;{ }^{* *} p<0.01$. 
Whether behavioural specificity will work in a similar way to increase compliance by health professionals is unclear. Focus group studies suggest that health professionals may see increased behavioural specificity and proceduralisation as prescriptive and a challenge to their professional autonomy. ${ }^{22}$ The successful implementation of guidelines aimed at health professionals will require a balance between standardising practice through increased specificity and allowing flexibility so that healthcare professionals can use clinical judgement where necessary.

This approach seeks to integrate relevant psychological evidence and theory into research aimed at developing effective implementation strategies in the development of clinical guidelines. ${ }^{23}{ }^{24}$ The findings need to be replicated in other groups of service users and clinicians, and in situations in which actual implementation-as well as intended implementation—can be measured.

\section{Authors' affiliations}

S Michie, K Lester, Centre for Outcomes Research and Effectiveness, Department of Psychology, University College London, UK

Competing interests: none

\section{REFERENCES}

1 Grimshaw J, Shirran L, Thomas R, et al. Changing provider behaviour: an overview of systematic reviews of interventions. Med Care 2001;39:112-45.

2 National Institute for Clinical Excellence. Treating and managing schizophrenia (core interventions): understanding NICE guidanceinformation for people with schizophrenia, their advocates and carers, and the public. London: National Institute for Clinical Excellence, 2002.

3 Clinical Guidelines Education Team. Implementing clinical guidelines: a resource for the healthcare team. Edinburgh: Balliere Tindall, 2001.

4 Michie S, Johnston M. Changing clinical behaviour by making guidelines specific. BMJ 2004;328:343-5.
5 Grol R, Dalhuijsen J, Thomas S, et al. Attributes of clinical guidelines that influence use of guidelines in general practice: observational study. BMJ 1998;317:858-61.

6 Jackson R, Feder G. Guidelines for clinical guidelines. BMJ 1998;317:427-8.

7 Ley P. Communicating with patients. London: Chapman \& Hall, 1998.

8 Ley P. Written communication. In: Baum A, Newman S, Weinman J, West R, McManus C, eds. Cambridge handbook of psychology, health and medicine. Cambridge: Cambridge University Press, 1997

9 Sheeran P, Milne S, Webb TL, et al. Implementation intentions. In: Conner M, Norman P, eds. Predicting health behaviour:research and practice with social cognition models, 2nd edn. Buckingham, UK: Open University Press, 1997.

10 Leventhal $H$, Singer R, Jones S. Effects of fear and specificity of recommendation upon attitudes and behaviour. J Pers Soc Psychol 1965;2:20-9.

11 Leventhal H, Watts J, Pagano F. Effects of fear and instructions on how to cope with danger. J Pers Soc Psychol 1967;6:313-21.

12 Kazdin A. Behaviour modification in applied settings. Belmont, CA: Thomson Learning, 2001

13 Plain English Campaign. How to write in plain English: 1979-2004, http:// www.plainenglish.co.uk/plainenglishguide.html.

14 Ajzen I. The theory of planned behaviour. Organ Behav Hum Perform 1991;50:179-211.

15 National Survey Programme. Local Health Services Questionnaire, Primary Care Trust Questionnaire, 2003.

16 Coulter A, Entwistle V, Gilbert D. Informing patients: an assessment of the quality of patient information materials. London: King's Fund, 1998.

17 Wittenbraker J, Gibbs B, Kahle L. Seat belt attitudes, habits and behaviours: an adaptive amendment to the Fishbein model. J Appl Soc Psychol 1983;13:406-21.

18 Cohen J. Statistical power analysis for the behavioural sciences, 2nd edn New York: Academic Press, 1988.

19 Schafer J. Monographs on statistics and applied probability: analysis of incomplete multivariate data. London: Chapman \& Hall, 1997.

20 Chan Y. Basic Statistics for doctors. Singapore Med J 2003:44:280-5.

21 Madden T, Ellen P, Ajzen I. A comparison of the theory of planned behaviour and the theory of reasoned action. Pers Soc Psychol Bull 1992;18:3-9.

22 Lawton R, Parker D. Procedures and the professional: the case of the British NHS. Soc Sci Med 1999:48:353-61.

23 Eccles MP, Grimshaw JM, Johnston M, et al. Health psychology models in implementation research: planned investigations, European Health Psychology Society, St Andrews, 2001.

24 Michie, S, Johnston, M, Abraham, C, et al. Making psychological theory useful for implementing evidence based practice: a consensus approach. Qual Saf Health Care 2005; 14:26-33. 